\title{
Equivalent Norms for Polynomials on the Sphere
}

\section{Jordi Marzo and Joaquim Ortega-Cerdà ${ }^{1}$}

${ }^{1}$ Departament de Matemàtica Aplicada i Anàlisi Universitat de Barcelona, Gran via 585, 08071 Barcelona, Spain

Correspondence to be sent to: jmarzo@mat.ub.es

We find necessary and sufficient conditions for a sequence of sets $E_{L} \subset \mathbb{S}^{d}$ in order to obtain the inequality

$$
\int_{\mathbb{S}^{d}}\left|Q_{L}\right|^{p} d \mu \leq C_{p} \int_{E_{L}}\left|Q_{L}\right|^{p} d \mu, \quad \forall L \geq 0,
$$

where $1 \leq p<+\infty, Q_{L}$ is any polynomial of degree smaller or equal than $L, \mu$ is a doubling measure, and the constant $C_{p}$ is independent of $L$. From this description, it follows an uncertainty principle for functions in $L^{2}\left(\mathbb{S}^{d}\right)$. We also consider weighted uniform versions of this result.

\section{Introduction}

The classical Logvinenko-Sereda theorem describes some equivalent norms for functions in the Paley-Wiener space $P W_{\Omega}^{p}$, i.e. functions in $L^{p}\left(\mathbb{R}^{d}\right)$ whose Fourier transform is supported in a prefixed bounded set $\Omega \subset \mathbb{R}^{d}$.

Theorem (Logvinenko-Sereda). Let $\Omega$ be a bounded set and let $1 \leq p<+\infty$. A set $E \subset \mathbb{R}^{d}$ satisfies

$$
\int_{\mathbb{R}^{d}}|f(x)|^{p} d x \leq C_{p} \int_{E}|f(x)|^{p} d x, \quad \forall f \in P W_{\Omega^{\prime}}^{p}
$$

Received March 27, 2007; Revised October 23, 2007; Accepted December 4, 2007

Communicated by Prof. Nikolai Makarov

(C) The Author 2008. Published by Oxford University Press. All rights reserved. For permissions,

please e-mail: journals.permissions@oxfordjournals.org. 
if and only if there is a cube $K \subset \mathbb{R}^{d}$, such that

$$
\inf _{x \in \mathbb{R}^{d}}|(K+x) \cap E|>0 .
$$

For a proof, see [4, pp. 112-6] or the original [5].

Comparison norms results of this kind are known in other contexts, see [4] and references therein for further information. The purpose of the present paper is to prove similar comparison results for $L^{p}$ norms of polynomials on the unit sphere $\mathbb{S}^{d}$.

In what follows, $\sigma$ will denote the surface measure in $\mathbb{S}^{d}$. We will prove the following theorem.

Theorem. Let $1 \leq p<\infty$. A sequence of sets $\mathcal{E}=\left\{E_{L}\right\}_{L \geq 0}$ in $\mathbb{S}^{d}$ satisfies

$$
\int_{\mathbb{S}^{d}}\left|Q_{L}\right|^{p} d \sigma \leq C_{p} \int_{E_{L}}\left|Q_{L}\right|^{p} d \sigma, \quad \forall L \geq 0,
$$

where $Q_{L}$ is any polynomial of degree smaller or equal than $L$ and the constant $C_{p}$ is independent of $L$, if and only if

$$
\inf _{L \in \mathbb{N}, 1-|z|=1 / L} h_{z}\left(E_{L}\right)>0,
$$

where $h_{z}(F)$ is the harmonic extension of $\chi_{F}$ to a point $z$ in the interior of the ball.

A more general (and precise) version will be stated and proved later on, see Theorem 1.5 once we have introduced some definitions and notation.

From this theorem, it follows an uncertainty principle for functions in $L^{2}\left(\mathbb{S}^{d}\right)$. For any $f \in L^{2}\left(\mathbb{S}^{d}\right)$, we have the spherical harmonics expansion $f=\sum_{\ell \geq 0} P_{\ell}(f)$, where $P_{\ell}$ is the orthogonal projection from $L^{2}\left(\mathbb{S}^{d}\right)$ onto the space of spherical harmonics of degree $\ell$.

Corollary 1.1. For a set $E \subset \mathbb{S}^{d}$, let $\delta=\inf _{1-|z|=1 / L} h_{z}(E)$. There exists a constant $C>0$ depending only on $\delta$, such that for any $f \in L^{2}\left(\mathbb{S}^{d}\right)$,

$$
\int_{\mathbb{S}^{d}}|f(u)|^{2} d \sigma(u) \leq C\left(\int_{E}|f(u)|^{2} d \sigma(u)+\sum_{\ell>L}\left\|P_{\ell}(f)\right\|^{2}\right) .
$$

The proof of the corollary amounts to show that (2) is equivalent to the inequality (1) and it can be found in [4, 3.1.1.A, pp. 88-9]. 


\subsection{Preliminaries and statements}

In $\mathbb{S}^{d}$, we take the geodesic distance

$$
d(u, v)=\arccos \langle u, v\rangle, \quad u, v \in \mathbb{S}^{d},
$$

and let $B(\omega, \delta) \subset \mathbb{S}^{d}$ denote the geodesic ball of center $\omega \in \mathbb{S}^{d}$ and radius $\delta>0$. We will denote by $\mathbb{B}(x, \delta)$ the ball of center $x \in \mathbb{R}^{d+1}$ with respect to the Euclidean metric in $\mathbb{R}^{d+1}$.

Let $\mathcal{H}_{\ell}$ be the spherical harmonics of degree $\ell$, i.e. the restrictions to the unit sphere $\mathbb{S}^{d}$ of the homogeneous harmonic polynomials in $d+1$ variables of degree $\ell$. Let $\Pi_{L}=\operatorname{span} \bigcup_{\ell=0}^{L} \mathcal{H}_{\ell}$ denote the spherical harmonics of degree less or equal than $L$. Observe that the restriction to $\mathbb{S}^{d}$ of any polynomial in $d+1$ variables of degree $\leq L$ belongs to $\Pi_{L}$.

In the Hilbert space $L^{2}(\sigma)$, let us denote by $Y_{\ell}^{1}, \ldots, Y_{\ell}^{h_{\ell}}$ an orthonormal basis of $\mathcal{H}_{\ell}$. Taking all these bases for $\ell=0, \ldots L$ together, we get an orthonormal basis for $\Pi_{L}$.

It is well known that the reproducing kernel for $\Pi_{L}$ is

$$
K_{L}(u, v)=\sum_{\ell=0}^{L} \sum_{j=1}^{h_{\ell}} Y_{\ell}^{j}(u) \overline{Y_{\ell}^{j}(v)}, \quad u, v \in \mathbb{S}^{d},
$$

and this expression does not depend on the choice of the bases. Using the ChristoffelDarboux formula (see, for instance, [7]), we obtain

$$
K_{L}(u, v)=\frac{\kappa_{d, L}}{\sigma\left(\mathbb{S}^{d}\right)} P_{L}^{(d / 2, d / 2-1)}(\langle u, v\rangle)
$$

where $P_{L}^{(\alpha, \beta)}$ stands for the Jacobi polynomial of degree $L$ and index $(\alpha, \beta)$ and $\kappa_{d, L} \sim L^{d / 2}$, as $L \rightarrow \infty$. (Here and in what follows, $\sim$ means that the ratio of the two sides is bounded from above and from below by two positive constants.) From now on, we denote $\lambda=$ $(d-2) / 2$.

Finally, we recall an estimate [11, p. 198], that will be used later on:

$$
P_{L}^{(1+\lambda, \lambda)}(\cos \theta)=\frac{k(\theta)}{\sqrt{L}}\left\{\cos \left((L+\lambda+1) \theta-\frac{(d+1) \pi}{4}\right)+\frac{O(1)}{L \sin \theta}\right\},
$$

if $c / L \leq \theta \leq \pi-(c / L)$, where

$$
k(\theta)=\pi^{-1 / 2}\left(\sin \frac{\theta}{2}\right)^{-\lambda-3 / 2}\left(\cos \frac{\theta}{2}\right)^{-\lambda-1 / 2} .
$$


Definition 1.2. We say that a measure $\mu$ is doubling if there exists a constant $C>0$, such that for any $u \in \mathbb{S}^{d}$ and any $\delta>0$,

$$
\mu(B(u, 2 \delta)) \leq C \mu(B(u, \delta))
$$

For such a measure, $\sup _{u, \delta} \mu(B(u, 2 \delta)) / \mu(B(u, \delta))$ is called the doubling constant of $\mu$.

It can be seen (see, for instance, [8, Lemma 2.1.]) that for $\mu$ doubling, there exists a $\gamma>0$, such that for $r>r^{\prime}$,

$$
\left(\frac{r}{r^{\prime}}\right)^{1 / \gamma} \lesssim \frac{\mu(B(u, r))}{\mu\left(B\left(u, r^{\prime}\right)\right)} \lesssim\left(\frac{r}{r^{\prime}}\right)^{\gamma}
$$

with constants depending only on the doubling constant of $\mu$.

Mimicking the Euclidean situation, we define the following concept.

Definition 1.3. Let $1 \leq p<\infty$ and let $\mu$ be a doubling measure. We say that the sequence of sets $\mathcal{E}=\left\{E_{L}\right\}_{L \geq 0} \subset \mathbb{S}^{d}$ is $L^{p}(\mu)$-Logvinenko-Sereda, if there exists a constant $C_{p}>0$, such that for any $Q \in \Pi_{L}$ and any $L$,

$$
\int_{\mathbb{S}^{d}}|Q(u)|^{p} d \mu(u) \leq C_{p} \int_{E_{L}}|Q(u)|^{p} d \mu(u) .
$$

Definition 1.4. The sequence of sets $\mathcal{E}=\left\{E_{L}\right\}_{L \geq 0} \subset \mathbb{S}^{d}$ is $\mu$-relatively dense, if there exist $r>0$ and $\varrho>0$, such that

$$
\inf _{u \in \mathbb{S}^{d}} \frac{\mu\left(E_{L} \cap B(u, r / L)\right)}{\mu(B(u, r / L))} \geq \varrho>0,
$$

for all $L$. When $\mu$ is the Lebesgue measure, we say that $\mathcal{E}$ is relatively dense.

Now we can state our main result.

Theorem 1.5. Let $\mathcal{E}=\left\{E_{L}\right\}_{L \geq 0}$ be a sequence of sets in $\mathbb{S}^{d} \cdot \mathcal{E}$ is $L^{p}(\mu)$-Logvinenko-Sereda for some $1 \leq p<\infty$ and $\mu$ a doubling measure, if and only if $\mathcal{E}$ is $\mu$-relatively dense.

If $\mu$ is absolutely continuous with an $A_{\infty}$ weight, it is possible to reformulate the $\mu$-relatively density in terms of the harmonic extension.

For a weight $\omega \geq 0$ in $\mathbb{S}^{d}$, we denote

$$
\omega(E)=\int_{E} \omega(u) d \sigma(u), \quad E \subset \mathbb{S}^{d} .
$$


Definition 1.6. A weight $\omega$ belongs to $A_{\infty}$ if there exist constants $B, \beta>0$, such that for any $E \subset B(u, \delta)$ measurable,

$$
\omega(B(u, \delta)) \leq B\left(\frac{\sigma(B(u, \delta))}{\sigma(E)}\right)^{\beta} \omega(E) .
$$

It is well known that that an $A_{\infty}$ weight defines a doubling measure, but the converse is not true, see [3].

Remark. It is clear by (7) that if $\mathcal{E}$ is relatively dense, it has to be $\omega$-relatively dense, but one can change condition (7) by

$$
\omega(E) \leq B\left(\frac{\sigma(E)}{\sigma(B(u, \delta))}\right)^{\beta} \omega(B(u, \delta)),
$$

see [10, Chapter. V, 1.7], therefore, to be relatively dense is equivalent to the same condition for the measure defined with $\omega \in A_{\infty}$.

We recall that for $x \in \mathbb{R}^{d+1}$ with $|x|<1$, the harmonic measure of subset $F \subset \mathbb{S}^{d}$ with respect to $x$ is

$$
h_{X}(F)=\frac{1}{\sigma\left(\mathbb{S}^{d}\right)} \int_{F} \frac{1-|x|^{2}}{|x-u|^{d+1}} d \sigma(u)=\frac{1}{\sigma\left(\mathbb{S}^{d}\right)} \int_{F} P(x, u) d \sigma(u),
$$

and $P(x, u)$ is the Poisson kernel in $\mathbb{S}^{d}$. The next result is a version for $\mathbb{S}^{d}$ of the one proved in [4, p. 114]. From now on, we will denote as $N=(0, \ldots, 0,1) \in \mathbb{R}^{d+1}$.

Lemma 1.7. The sequence $\left\{E_{L}\right\}_{L \geq 0} \subset \mathbb{S}^{d}$ is relatively dense, if and only if there exists $\alpha>0$, such that

$$
h_{X}\left(E_{L}\right) \geq \alpha, \text { for all } x \in \mathbb{R}^{d+1} \text { with }|x|=1-1 / L .
$$

Proof. Observe that both conditions are rotation invariant. For $u$, such that $d(u, N)<$ $r / L$, we have $C L^{d} \leq P(|x| N, u) \leq 2 L^{d}$, where $C>0$ is a constant depending on $r$ and $d$. For $\theta=d(u, N)>r / L$,

$$
P(|X| N, u) \lesssim \frac{\frac{2}{L}-\frac{1}{L}}{\sin ^{d+1} \frac{\theta}{2}} \lesssim \frac{L^{d}}{r^{d+1}}
$$

These bounds are all we need to prove the result. In one direction,

$$
h_{|X| N}\left(E_{L}\right) \gtrsim L^{d} \sigma\left(E_{L} \cap B(N, r / L)\right) \gtrsim \varrho>0 .
$$


Conversely,

$$
\begin{aligned}
\sigma\left(\mathbb{S}^{d}\right) \alpha & \leq \int_{E_{L}} P(|x| N, u) d \sigma(u) \leq \int_{E_{L}}\left(\chi_{B(N, r / L)}(t)+\chi_{B(N, r / L)^{c}}(t)\right) P(|x| N, u) d \sigma(u) \\
& \leq 2 L^{d} \sigma\left(E_{L} \cap B(N, r / L)\right)+\sum_{\log _{2} \pi L / r \geq j \geq 0} \int_{\frac{2 j_{r}}{L}<d(u, N)<\frac{2^{j+1_{r}}}{L}} \chi_{E_{L}}(u) P(|x| N, u) d \sigma(u) \\
& \leq C_{r} \frac{\sigma\left(E_{L} \cap B(N, r / L)\right)}{\sigma(B(N, r / L))}+\frac{C}{r} \sum_{j \geq 0} \frac{1}{2^{d j}} .
\end{aligned}
$$

Taking $r>0$ big enough, we get the result.

Remark. We have proved that there exist $r, \varrho$, such that $\sigma\left(E_{L} \cap B(u, r / L)\right) \geq \varrho \sigma(B(u, r / L))$ for $L$ big enough, if and only if there exists $\alpha$, such that $h_{(1-1 / L) u}\left(E_{L}\right) \geq \alpha$. This new formulation depends only on one parameter.

In Theorem 1.5, when the dimension $d=1$, there are already some results known. In this case, it is possible to replace polynomials by holomorphic polynomials. If, moreover, $\mu$ is the Lebegue measure, the space of holomorphic polynomials can be seen as a model space, so Volberg result [12] extending the original theorem of Logvinenko and Sereda to model spaces apply. Also, when $d=1$ and the measure $\mu$ is an $A_{\infty}$ weight, the sufficiency of condition (6) was proved in [8, Theorem 5.4].

Condition (6) is true for some $\omega \in A_{\infty}$, if and only if it is true for the Lebesgue measure $\sigma$. So, we have comparison of norms for any $\omega \in A_{\infty}$, if and only if we have (6) for the Lebesgue measure $\sigma$. The discussion following [8, Theorem 5.4] shows that this is not true for arbitrary doubling measures.

To the best of our knowledge, for dimensions greater than one, Theorem 1.5 is new, even in the case of Lebesgue measure.

The outline of this paper is as follows. In Section 2, we will prove Theorem 1.5.

In Section 3, we deal with the uniform norm case. In this setting, we have an analogous result to Proposition 2.2, namely Theorem 3.1. To consider weighted versions of this result, an obvious requirement is to take weights bounded above. We take the reverse Hölder class $R H_{\infty}$ of those weights, satisfying reverse Hölder inequalities in a uniform way. This class that was also introduced in [8] for the one-dimensional case, is shown to be optimal in a certain sense.

\section{Main Results}

Proposition 2.1. Let $1 \leq p<\infty, \mu$ be a doubling measure, and let $\mathcal{E}=\left\{E_{L}\right\}_{L \geq 0}$ be a sequence of sets in $\mathbb{S}^{d}$. If $\mathcal{E}$ is $L^{p}(\mu)$-Logvinenko-Sereda, then it is $\mu$-relatively dense. 
Proof. We focus on $d \geq 2$, but only minor changes will prove the one-dimensional case. The strategy is to apply the $L^{p}(\mu)$-comparison of norms to a power of the reproducing kernel and to use classical estimates on the Jacobi polynomials.

Let $Q(v)=\left(P_{L}^{(1+\lambda, \lambda)}(\langle v, N\rangle)\right)^{\ell} \in \Pi_{\ell L}$ and let $0<r<<R$. We have by hypothesis,

$$
\begin{aligned}
\int_{B(N, r / L)}|Q(v)|^{p} d \mu(v) & \leq \int_{\mathbb{S}^{d}}|Q(v)|^{p} d \mu(v) \leq C \int_{E_{\ell L}}|Q(v)|^{p} d \mu(v) \\
& \lesssim \int_{E_{\ell L} \cap B(N, R / L)}|Q(v)|^{p} d \mu(v)+\int_{\mathbb{S}^{d} \backslash B(N, R / L)}|Q(v)|^{p} d \mu(v) .
\end{aligned}
$$

Observe that $Q$ reaches its maximum in $N$, [11] so applying Bernstein's inequality to the polynomial restricted to a great circle, we get for any $v$, such that $d(v, N)<$ $r / L$

$$
|Q(v)-Q(N)| \leq|Q(N)| \ell r
$$

Therefore, for $r$ small enough, we have $|Q(v)|^{p} \sim|Q(N)|^{p}$, if $d(v, N)<r / L$. We can bound the integral in the left hand side of (8) as

$$
\int_{B(N, r / L)}|Q(v)|^{p} d \mu(v) \gtrsim\left(P_{L}^{(1+\lambda, \lambda)}(1)\right)^{p \ell} \mu(B(N, r / L)) \sim L^{\frac{p \ell d}{2}} \mu(B(N, r / L)) .
$$

Since $\left|P_{L}^{(1+\lambda, \lambda)}(\cos \theta)\right| \lesssim L^{\lambda}$ for $\pi-\frac{R}{L} \leq \theta \leq \pi$,

$$
\begin{aligned}
L^{\frac{p \ell d}{2}} \mu(B(N, r / L)) \lesssim & L^{\frac{p \ell d}{2}} \mu\left(E_{\ell L} \cap B(N, R / L)\right)+L^{\frac{p \ell(d-2)}{2}} \mu(B(S, R / L)) \\
& +\int_{R / L<d(v, N)<\pi-R / L}|Q(v)|^{p} d \mu(v) .
\end{aligned}
$$

To control the last integral, we may use Szegö estimate (3)

$$
\begin{aligned}
\int_{R / L<d(v, N)<\pi-R / L}|Q(v)|^{p} d \mu(v) \lesssim & L^{-p \ell / 2} \int_{R / L<d(v, N)<\pi / 2}\left|\sin ^{d+1} \frac{d(v, N)}{2}\right|^{-\ell p / 2} d \mu(v) \\
& +L^{-p \ell / 2} \int_{\pi / 2<d(v, N)<\pi-R / L}\left|\cos ^{d-1} \frac{d(v, N)}{2}\right|^{-\ell p / 2} d \mu(v) \\
= & I+I I .
\end{aligned}
$$

For part $I$, we take $\ell$ big enough to get $C(\mu)<2^{p l(d+1) / 4}$, where $C(\mu)$ is the doubling constant of $\mu$. We split the sphere in diadic "bands" around the north pole and using the 
doubling property for $\mu$, we get

$$
\begin{aligned}
L^{p \ell / 2} I & \lesssim \int_{R / L<d(v, N)} \frac{1}{d(v, N)^{(d+1) l p / 2}} d \mu(v) \leq \sum_{J \geq j \geq 0} \int_{2^{j} R / L<d(v, N)<2^{j+1} R / L} \frac{d \mu(v)}{\left(2^{j} R / L\right)^{(d+1) l p / 2}} \\
& \leq \sum_{J \geq j \geq 0} \frac{\mu\left(B\left(N, 2^{j+1} R / L\right)\right)}{\left(2^{j} R / L\right)^{(d+1) l p / 2}} \leq \frac{\mu(B(N, R / L))}{(R / L)^{(d+1) l p / 2}} \sum_{j \geq 0}\left(\frac{C(\mu)}{2^{\alpha+\lambda}}\right)^{j} \lesssim \frac{\mu(B(N, R / L))}{(R / L)^{(d+1) l p / 2}}
\end{aligned}
$$

where $\mathbb{N} \ni J \geq \log _{2}(\pi L / R)$.

For part II, the same computation taking dyadic "bands" around the south pole shows that

$$
L^{p \ell / 2} I I \lesssim \frac{\mu(B(S, R / L))}{(R / L)^{(d-1) l p / 2}} \lesssim \mu(B(S, R / L)) L^{(d-1) l p / 2}
$$

We use now property (4) and the $\gamma$ given there for $\mu$ to estimate $\mu(B(S, R / L))$. If we put all estimates together and for $\ell$ big enough, we get

$$
\begin{aligned}
\mu(B(N, r / L)) & \lesssim \mu\left(E_{\ell L} \cap B(N, R / L)\right)+L^{-p \ell} \mu(B(S, R / L))+\frac{\mu(B(N, R / L))}{R^{(d+1) l p / 2}} \\
& \lesssim \mu\left(E_{\ell L} \cap B(N, R / L)\right)+\frac{R^{\gamma}}{L^{\gamma+p \ell}}+\left(\frac{R}{r}\right)^{\gamma} \frac{\mu(B(N, r / L))}{R^{(d+1) l p / 2}} .
\end{aligned}
$$

As $\mu(B(N, r / L)) \geq(r / L)^{1 / \gamma}$, the second term is $o(\mu(B(N, r / L)))$ when $L \rightarrow \infty$ for $\ell$ big enough. For the third term, we choose $\ell$, such that $(R / r)^{\gamma} \leq R^{(d+1) l p / 2} / 2$. Thus, picking $\ell$ big enough, we have proved that

$$
\mu(B(N, r / L)) \lesssim \mu\left(E_{\ell L} \cap B(N, R / L)\right), \quad \text { if } L \geq L_{0}
$$

Of course, the constants do not depend on the center of the balls being the north pole. Moreover, by the doubling property, $\mu(B(N, R / L)) \simeq \mu(B(N, r / L))$. By choosing a bigger $R$, we get

$$
\mu(B(z, R / L)) \lesssim \mu\left(E_{\ell L} \cap B(z, R / L)\right), \quad \forall z \in \mathbb{S}^{d}, \quad L \geq 0
$$

Finally, we have only controlled the density of the sequence of sets $\left\{E_{l L}\right\}_{L \geq 0}$. But, we could have used the same argument to the sequence $\mathcal{E}^{\prime}=\left\{E_{L+1}\right\}_{L \geq 0}$ from the very beginning and we will then obtain a control of the density the sets $\left\{E_{l L+1}\right\}_{L \geq 0}$. By repeating the argument $l$ times, we get the desired result. 
Remark. The somehow simpler polynomials

$$
\left(\frac{1+\langle v, N\rangle}{2}\right)^{L \ell} \text { or }\left(\frac{1-\langle v, N\rangle^{L+1}}{(L+1)(1-\langle v, N\rangle)}\right)^{\ell}
$$

that peak at $N$ and have been considered in other contexts do not decrease fast enough near the pole north to be chosen as test functions for the comparison of norms, as we did with the polynomial $Q$ above.

Proposition 2.2. If $\left\{E_{L}\right\}_{L \geq 0}$ is $\mu$-relatively dense for some doubling measure $\mu$, then it is $L^{p}(\mu)$-Logvinenko-Sereda for any $1 \leq p<\infty$.

Proof. We consider a regularized version of $\mu$

$$
\mu_{L}(u)=\frac{\mu(B(u, 1 / L))}{\sigma(B(u, 1 / L))}, \quad L \geq 0 .
$$

By Corollary 3.4. in [2], we have

$$
\int_{\mathbb{S}^{d}}\left|Q_{L}(u)\right|^{p} d \mu(u) \sim \int_{\mathbb{S}^{d}}\left|Q_{L}(u)\right|^{p} \mu_{L}(u) d \sigma(u), \quad Q_{L} \in \Pi_{L}
$$

The regularization of $\mu$ is pointwise equivalent to a polynomial. Indeed, there exists $R_{L} \in \Pi_{L}$ nonnegative, such that for any $u \in \mathbb{S}^{d}$

$$
\mu_{L}(u) \sim R_{L}(u)^{p}
$$

with constant depending only on $d$, the doubling constant for $\mu$ and $p$, see [2, Lemma 4.6]. Given $Q_{L} \in \Pi_{L}$, let $M_{2 L} \in \Pi_{2 L}$, such that $M_{2 L}=Q_{L} R_{L}$ in $\mathbb{S}^{d}$. Following an idea of D. H. Luecking [6], we consider, for $\epsilon>0$ and $r>0$, the set of points $z \in \mathbb{S}^{d}$, such that $M_{2 L}(z)$ has the same size as its average, i.e.

$$
A=A_{\epsilon, r, M_{2 L}}=\left\{z \in \mathbb{S}^{d}:\left|M_{2 L}(z)\right|^{p} \geq \epsilon f_{\mathbb{B}(z, r / L)}\left|M_{2 L}(u)\right|^{p} d m(u)\right\} .
$$

Most of the norm of $M_{2 L}$ is concentrated on $A$,

$$
\begin{aligned}
\int_{\mathbb{S}^{d} \backslash A}\left|M_{2 L}(z)\right|^{p} d \sigma(z) & \leq \epsilon \int_{\mathbb{S}^{d} \backslash A}\left(f_{\mathbb{B}(z, r / L)}\left|M_{2 L}(u)\right|^{p} d m(u)\right) d \sigma(z) \\
& \leq \epsilon \int_{|1-| u||<r / L}\left|M_{2 L}(u)\right|^{p}\left(\int_{\mathbb{S}^{d} \backslash A} \frac{\chi_{\mathbb{B}(z, r / L)}(u)}{m(\mathbb{B}(z, r / L))} d \sigma(z)\right) d m(u) \\
& \lesssim \epsilon L \int_{|1-| u||<r / L}\left|M_{2 L}(u)\right|^{p} d m(u) \sim \epsilon \int_{\mathbb{S}^{d}}\left|M_{2 L}(z)\right|^{p} d \sigma(z),
\end{aligned}
$$

using [7, Corollary 4.3] in the last estimate, the constants are independent of $L$. 
Thus, it is enough to show that

$$
\int_{A}\left|M_{2 L}(u)\right|^{p} d \sigma(u) \lesssim \int_{E_{L}}\left|Q_{L}(u)\right|^{p} d \mu(u) .
$$

All we need to prove is the existence of a constant $C>0$, such that for all $\omega \in A$,

$$
\left|Q_{L}(\omega)\right|^{p} \leq \frac{C}{\mu(B(\omega, r / L))} \int_{B(\omega, r / L) \cap E_{L}}\left|Q_{L}(u)\right|^{p} d \mu(u) .
$$

Indeed, if this is the case then

$$
\begin{aligned}
\int_{A}\left|M_{2 L}(\omega)\right|^{p} d \sigma(\omega) & \leq C \int_{E_{L}}\left|Q_{L}(u)\right|^{p} \int_{\mathbb{S}^{d}} \frac{\chi_{B(\omega, r / L)}(u)}{\mu(B(\omega, r / L))} \mu_{L}(\omega) d \sigma(\omega) d \mu(u) \\
& \lesssim \int_{E_{L}}\left|Q_{L}(u)\right|^{p} d \mu(u) .
\end{aligned}
$$

To prove (9), we argue by contradiction. If (9) is false, there are for any $n \in \mathbb{N}$ polynomials $Q_{n} \in \Pi_{L_{n}}$ and $\omega_{n} \in A$, such that

$$
\left|Q_{n}\left(\omega_{n}\right)\right|^{p}>\frac{n}{\mu\left(B\left(\omega_{n}, r / L_{n}\right)\right)} \int_{B\left(\omega_{n}, r / L_{n}\right) \cap E_{L_{n}}}\left|Q_{n}(u)\right|^{p} d \mu(u) .
$$

Since $\mu$ is doubling, then $R_{L_{n}}\left(\omega_{n}\right) \sim R_{L_{n}}(u)$ for any $u \in B\left(\omega_{n}, r / L_{n}\right)$. Let $M_{n} \in \Pi_{2 L_{n}}$, such that $M_{n}=Q_{n} R_{L_{n}}$ in $\mathbb{S}^{d}$

$$
\left|M_{n}\left(\omega_{n}\right)\right|^{p} \gtrsim \frac{n}{\mu\left(B\left(\omega_{n}, r / L_{n}\right)\right)} \int_{B\left(\omega_{n}, r / L_{n}\right) \cap E_{L_{n}}}\left|M_{n}(u)\right|^{p} d \mu(u) .
$$

By means of a rotation, a dilation, and a translation, we send $\omega_{n}$ to the origin in $\mathbb{R}^{d+1}$, the ball $\mathbb{B}\left(\omega_{n}, r / L_{n}\right)$ to $\mathbb{B}(0,1) \subset \mathbb{R}^{d+1}$, and the set $E_{L_{n}}$ to

$$
E_{n} \subset \partial \mathbb{B}\left(-\left(L_{n} / r\right) N, L_{n} / r\right) \cap \mathbb{B}(0,1)
$$

The composition of these applications with our harmonic polynomials $M_{n}$ are harmonic functions $f_{n}$ that, after normalization, we can assume that satisfy

$$
\int_{\mathbb{B}(0,1)}\left|f_{n}\right|^{p} d m=1
$$

The subharmonicity of $\left|f_{n}\right|^{p}$ and the fact that $\omega_{n} \in A$ tells us that

$$
\epsilon \lesssim\left|f_{n}(0)\right|^{p} \lesssim 1
$$

and this property together with (10) yields

$$
\frac{1}{n} \gtrsim \int_{\mathbb{B}(0,1) \cap E_{n}}\left|f_{n}(u)\right|^{p} d \mu_{n}(u),
$$




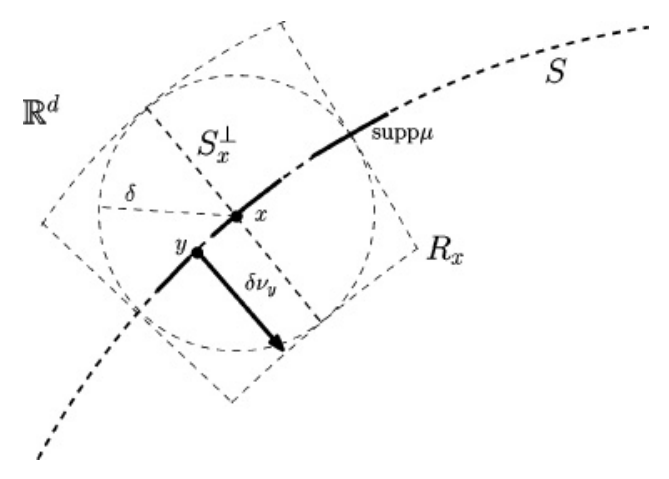

Fig. 1. Construction of the measures $v_{n}$.

where $\mu_{n}$ is the push forward of the measure $\mu$, supported in $\partial \mathbb{B}\left(-\left(L_{n} / r\right) N, L_{n} / r\right) \cap \mathbb{B}(0,1)$, and normalized in such a way that $\mu_{n}(\mathbb{B}(0,1))=1$.

We have that $\left\{f_{n}\right\}$ is a normal family in $\mathbb{B}(0,1)$ and therefore, there exists a subsequence that converges locally uniformly on $\mathbb{B}$ to an harmonic function that we call $f$.

We observe that the relative density hypothesis yields

$$
\inf _{n} \mu_{n}\left(E_{n} \cap \mathbb{B}(0,1)\right)>0 .
$$

Let $\tau$ be a weak- $*$ limit of a subsequence of $\tau_{n}=\mu_{n} \chi_{E_{n}}$, having supp $\tau \subset \mathbb{R}^{d} \times\{0\}$ and $\tau \not \equiv 0$. We will consider the measure $\tau_{n}$ that has support in $\partial \mathbb{B}\left(-\left(L_{n} / r\right) N, L_{n} / r\right) \cap \mathbb{B}(0,1)$ as having support in $\mathbb{R}^{d} \times\{0\}$. To do so, we define the measure $\tilde{\tau}_{n}$ as the "projection" of the measure $\tau_{n}$ to $\mathbb{R}^{d} \times\{0\}$, i.e. $\tilde{\tau}_{n}(A)=\tau_{n}(A \times[-1,1])$, for $A \subset \mathbb{R}^{d}$.

We observe that $f$ restricted to $\mathbb{R}^{d} \times\{0\}$ is real analytic. Condition (11) implies that $f=0 \tau$-a.e. and therefore, $\operatorname{supp} \tau \subset\left\{f_{\mathbb{R}^{d} \times\{0\}}=0\right\}$.

We want to show that $\operatorname{supp} \tau \subset \mathbb{R}^{d}$ (identifying $\mathbb{R}^{d} \times\{0\}$ and $\mathbb{R}^{d}$ ) cannot lie on a real analytic $(d-1)$-dimensional submanifold $S \subset \mathbb{R}^{d}$ (the worst case). We argue by contradiction. Let $x \in \operatorname{supp} \tau \subset S$ and $\delta>0$, such that $\tau(B(x, \delta))=\epsilon>0$.

We can consider for any $y \in B(x, \delta) \cap S$ the unitary vector $v_{Y}$ in $\mathbb{R}^{d}$ normal to $S$ in the point $y$ (see Figure 1) and define the "square" $B(x, \delta) \subset R_{X}$

$$
R_{X}=\left\{y+\eta v_{Y}: y \in B(x, \delta) \cap S,|\eta|<\delta\right\} .
$$

Now, we can define measures $v_{n}$ in $S_{x}^{\perp}=\left\{x+\eta v_{x}:|\eta|<\delta\right\}$ just by taking for $A \subset S_{x}^{\perp}$ the set $\tilde{A} \subset(-\delta, \delta)$, such that $x+\tilde{A} v_{x}=A$ and defining

$$
v_{n}(A)=\tilde{\tau}_{n}\left(\left\{y+\eta v_{Y} \in R_{X}: \eta \in \tilde{A}\right\}\right) .
$$


By hypothesis, $v_{n}$ converges vaguely to some nonzero measure $v$ with support in $\{x\}$, because $v_{n}\left(S_{X}^{\perp}\right)=\tilde{\tau}_{n}\left(R_{X}\right) \geq \tilde{\tau}_{n}(B(x, \delta)) \geq \epsilon>0$. To get a contradiction, it is enough to show that $v$ is dominated by a doubling measure in $S_{x}^{\perp}$.

We define

$$
\gamma_{n}(A)=\tilde{\mu}_{n}\left(\left\{y+\eta v_{Y} \in R_{X}: \eta \in \tilde{A}\right\}\right), \quad A \subset S_{x}^{\perp},
$$

where as before, $\tilde{\mu}_{n}$ is the "projection" of $\mu_{n}$ to $\mathbb{R}^{d}$. Observe that $v_{n}(A) \leq \gamma_{n}(A)$, and that $\gamma_{n}$ are doubling measures, all with the same doubling constant. Indeed, for any $\delta>\alpha>0$, there exist $y_{1}, \ldots, y_{N} \in B(x, \delta) \cap S$, such that

$$
\left\{y+\eta v_{Y} \in R_{X}:|\eta|<2 \alpha\right\} \subset \bigcup_{j=1}^{N} B\left(y_{j}, 5 \alpha / 2\right), \quad \sum_{j=1}^{N} \chi_{B\left(y_{j}, 5 \alpha / 4\right)} \leq C
$$

The "projection" of the $\mu_{n}$ to $\mathbb{R}^{d}$ are doubling measures, all with the same doubling constant, so

$$
\begin{aligned}
\gamma_{n}\left(x+(-2 \alpha, 2 \alpha) v_{x}\right) & \leq \sum_{j=1}^{N} \tilde{\mu}_{n}\left(B\left(y_{j}, 5 \alpha / 2\right)\right) \\
& \leq C \sum_{j=1}^{N} \tilde{\mu}_{n}\left(B\left(y_{j}, 5 \alpha / 4\right)\right) \leq C \gamma_{n}\left(x+(\alpha, \alpha) v_{X}\right) .
\end{aligned}
$$

Therefore, by (4), we have $C, \gamma>0$ constants, such that $v_{n}\left(x+(-r, r) v_{x}\right) \leq C r^{\gamma}$ and the same holds for $v$. Observe that $\operatorname{supp} v$ has to be of Hausdorff dimension $\geq \gamma>0$ and this would contradict $\operatorname{supp} v=\{x\}$.

\section{Uniform norm case}

In this section, we want to find sufficient conditions in the sequence $\mathcal{E}=\left\{E_{L}\right\}_{L \geq 0}$ in order to get the $L^{\infty}$-Logvinenko-Sereda property, i.e.

$$
\sup _{u \in \mathbb{S}^{d}}\left|Q_{L}(u)\right| \leq C \sup _{u \in E_{L}}\left|Q_{L}(u)\right|, \quad \text { for any } Q_{L} \in \Pi_{L},
$$

with $C$ a constant that does not depend on $L$.

Our main result is the following theorem. 
Theorem 3.1. If $\mathcal{E}$ is relatively dense, then it is $L^{\infty}$-Logvinenko-Sereda.

Remark. The converse is false, because there exist discrete sets (so, with zero Lebesgue measure) with comparison property (12).

In [8], the authors deal with the weighted one-dimensional case of Theorem 3.1. In this uniform case, it is a natural assumption to consider only bounded weights. They considered the family of weights $\omega \geq 0$, such that

$$
\omega(u) \leq \frac{C}{\sigma(B)} \int_{B} \omega(v) d \sigma(v),
$$

for any spherical cap $B \subset \mathbb{S}^{d}$ and $u \in B$. Following [1], we call $R H_{\infty}$ this family.

Definition 3.2. Let $\omega \geq 0$ be a function, such that property (13) holds for almost every $u \in \mathbb{S}^{d}$, we say that $\omega$ is in the reverse Hölder class $R H_{\infty}$.

To justify the name of this class, observe that for $\omega \in R H_{\infty}$, the reverse Hölder inequality

$$
\left(\frac{1}{\sigma(B)} \int_{B} \omega^{s}(u) d \sigma(u)\right)^{s} \leq \frac{C}{\sigma(B)} \int_{B} \omega(u) d \sigma(u), \quad B \subset \mathbb{S}^{d} \text { spherical cap }
$$

holds for each $s>1$, (i.e. $\omega \in R H_{s}$ ) and the best constant $C$ is bounded by the constant appearing in (13). And conversely, if the reverse Hölder inequality holds for each $s>1$ with a constant independent of $s$, then $\omega \in R H_{\infty}$, see [1].

Observe that $R H_{\infty} \subset A_{\infty}$. Roughly speaking, $\omega$ belongs to $A_{1}$, if and only if $1 / \omega \in$ $R H_{\infty}$. These weights can have high-order zeros in $\mathbb{S}^{d}$.

In this section, we will prove the one-dimensional unweighted result first and then extend it to $\mathbb{S}^{d}$. Using this unweighted case and adapting some results from $[2,8]$, we will prove the weighted result.

Proof. We start with the one-dimensional case. Using the Lemma 1.7, we get $h_{x}\left(E_{L}\right) \geq \alpha$, for any $|x|=1-1 / L$. Let $p$ be a polynomial of degree $L$, there exists a holomorphic polynomial $q$ of degree $2 L$, such that $|p|=|q|$ in $\mathbb{S}^{1}$. So, for any $x \in \mathbb{R}^{2}$ with $|x|=1-1 / L$,

$$
\begin{aligned}
\log |q(x)| & \leq h_{X}\left(E_{L}\right) \log \left(\max _{E_{L}}|q|\right)+h_{X}\left(\mathbb{S}^{1} \backslash E_{L}\right) \log \left(\max _{\mathbb{S}^{1}}|q|\right) \\
& =\log \|q\|_{\mathbb{S}^{1}}+h_{X}\left(E_{L}\right) \log \frac{\|q\|_{E_{L}}}{\|q\|_{\mathbb{S}^{1}}} \leq \log \|q\|_{\mathbb{S}^{1}}+\alpha \log \frac{\|q\|_{E_{L}}}{\|q\|_{\mathbb{S}^{1}}},
\end{aligned}
$$


because $\|q\|_{E_{L}} /\|q\|_{\mathbb{S}^{1}} \leq 1$ and so, $|q(x)| \leq\|p\|_{E_{L}}^{\alpha}\|p\|_{\mathbb{S}^{1}}^{1-\alpha}$. Finally, one can see that

$$
\max _{x \in \mathbb{S}^{1}}|q(x)| \leq C \max _{|x|=1-1 / L}|q(x)|
$$

where $C$ is independent of $L$, see [9, Lemma 2].

Now we consider the case $d>1$. Let $Q \in \Pi_{L}$ and suppose that $\max _{\mathbb{S}^{d}}|Q|=$ $|Q(N)|=1$. We have that,

$$
\frac{\sigma\left(E_{L} \cap B(N, r / L)\right)}{\sigma(B(N, r / L))} \geq \epsilon>0
$$

Denoting $\tilde{\omega}=(\omega, 0) \in \mathbb{R}^{d+1}$ for $\omega \in \mathbb{S}^{d-1}$, we have that $G_{\omega}(\theta)=N \cos \theta+\tilde{\omega} \sin \theta$ is a geodesic in $\mathbb{S}^{d}$, if $\theta \in[-\pi, \pi]$. Therefore, denoting $\mathbb{S}_{+}^{d-1}=\left\{\left(\omega_{1}, \ldots, \omega_{d}\right) \in \mathbb{S}^{d-1}: \omega_{d}>0\right\}$,

$$
\sigma\left(E_{L} \cap B(N, r / L)\right)=\int_{\mathbb{S}_{+}^{d-1}} \int_{-r / L}^{r / L} \chi_{E_{L}}\left(G_{\omega}(\theta)\right) \sin ^{d-1} \theta d \theta d \omega
$$

Now,

$$
\begin{aligned}
\epsilon \sigma(B(N, r / L)) & \leq \int_{\mathbb{S}_{+}^{d-1}} \int_{-r / L}^{r / L} \chi_{E_{L}}\left(G_{\omega}(\theta)\right) \sin ^{d-1} \theta d \theta d \omega \\
& \leq \int_{\mathbb{S}_{+}^{d-1}} \int_{-r / L}^{r / L} \chi_{E_{L}}\left(G_{\omega}(\theta)\right)\left(\frac{r}{L}\right)^{d-1} d \theta d \omega \\
& \leq\left(\frac{r}{L}\right)^{d-1} \int_{\mathbb{S}_{+}^{d-1}} \sigma\left(E_{L} \cap B_{\omega}(N, r / L)\right) d \omega
\end{aligned}
$$

where $B_{\omega}(N, r / L)=\left\{G_{\omega}(\theta):|\theta| \leq r / L\right\}$. We get

$$
\int_{\mathbb{S}_{+}^{d-1}} \frac{\sigma\left(E_{L} \cap B_{\omega}(N, r / L)\right)}{\sigma\left(B_{\omega}(N, r / L)\right)} d \omega \geq C_{d} \epsilon
$$

and therefore, there exists a direction $\omega \in \mathbb{S}_{+}^{d-1}$, such that

$$
\frac{\sigma\left(E_{L} \cap B_{\omega}(N, r / L)\right)}{\sigma\left(B_{\omega}(N, r / L)\right)} \geq C_{d} \epsilon>0 .
$$

Let $G_{\omega}^{*}=\left\{G_{\omega}(\theta): \theta \in[-\pi, \pi]\right\}, p\left(e^{i \theta}\right)=Q\left(G_{\omega}(\theta)\right)$ and $q$ be a holomorphic polynomial of degree at most $2 L$, such that $|p|=|q|$. By using Bernstein inequality, $\|q\|_{\mathbb{S}^{1}} \leq C \mid q(1-$ $1 / 4 L) \mid$, with $C$ a constant independent of $L$. Finally, as $h_{1-1 / 4 L}\left(E_{L} \cap G_{\omega}^{*}\right) \geq \alpha$, we may 
apply (14) to $x=1-1 / 4 L$ and we get,

$$
\|Q\|_{\mathbb{S}^{d}}=\|q\|_{\mathbb{S}^{1}} \leq C\|p\|_{E_{L} \cap G_{\omega}^{*}}^{\alpha}\|p\|_{\mathbb{S}^{1}}^{1-\alpha} \leq C\|Q\|_{E_{L}}^{\alpha}\|Q\|_{\mathbb{S}^{d}}^{1-\alpha} .
$$

Using Theorem 3.1, we prove the following weighted version.

Corollary 3.3. If $\mathcal{E}$ is relatively dense and $\omega \in R H_{\infty}$, then

$$
\sup _{u \in \mathbb{S}^{d}}\left|Q_{L}(u)\right| \omega(u) \leq C \sup _{u \in E_{L}}\left|Q_{L}(u)\right| \omega(u), \text { for any } Q_{L} \in \Pi_{L},
$$

with $C$ a constant that does not depend on $L$.

Remark. This result is optimal in some sense, because there are unbounded weights belonging to all reverse Hölder classes, i.e. in particular, $R H_{\infty} \varsubsetneqq \cap_{s>1} R H_{s}$, see [1, p. 2948].

Proof. By definition of $R H_{\infty}$ weight,

$$
\omega(u) \leq C \omega_{L}(u)=\frac{1}{\sigma(B(u, 1 / L))} \int_{B(u, 1 / L)} \omega(v) d \sigma(v) .
$$

Now, [2, Lemma 4.6] provide us with $R_{L} \in \Pi_{L}$ nonnegative, such that for any $u \in \mathbb{S}^{d}$ $\omega_{L}(u) \sim R_{L}(u)$, with constant depending only on the doubling constant for $\omega_{L}$.

Now we want to construct a relatively dense regularization of $E_{L}$ that we will denote $E_{L}^{*}$. Given $\epsilon>0$, let $V=V_{\epsilon, L} \subset \mathbb{S}^{d}$ discrete and such that,

$$
\mathbb{S}^{d} \subset \bigcup_{v \in V} B(v, \epsilon / L), \text { and } \sum_{v \in V} \chi_{B(v, \epsilon / L)}(u) \leq C_{d}, \quad u \in \mathbb{S}^{d}
$$

For $\delta>0$, that we will determine afterwards, let

$$
V_{g}=\left\{v \in V: \sigma\left(B(v, \epsilon / L) \cap E_{L}\right) \geq \delta \sigma(B(v, \epsilon / L))\right\}, \text { and } E_{L}^{*}=\bigcup_{v \in V_{g}} B(v, \epsilon / L)
$$


We denote $V_{b}=V \backslash V_{g}$. Let $V(u)$ be the set of those $v \in V$, such that $B(v, \epsilon / L) \cap B(u, r / L) \neq \emptyset$ and likewise, we split $V(u)=V_{g}(u) \cup V_{b}(u)$,

$$
\begin{aligned}
\sigma(B(u, r / 2 L)) & \leq \sigma\left(\bigcup_{v \in V_{g}(u)} B(v, \epsilon / L)\right)+\sigma\left(\bigcup_{v \in V_{b}(u)} B(v, \epsilon / L)\right) \\
& \leq \sigma\left(E_{L}^{*} \cap B(u, r / L)\right)+\sigma\left(\bigcup_{v \in V_{b}(u)} B(v, \epsilon / L)\right) .
\end{aligned}
$$

Using the relative density of $E_{L}$ and the property of being in $V_{b}$, we get

$$
\begin{aligned}
\varrho \sigma(B(u, r / 2 L)) & \leq \sigma\left(E_{L} \cap B(u, r / L)\right) \\
& \leq C_{d} \delta \sigma(B(u, r / L))+\sigma\left(E_{L} \cap\left(B(u, r / L) \backslash \bigcup_{v \in V_{b}(u)} B(v, \epsilon / L)\right)\right)
\end{aligned}
$$

so for $\delta$ small enough,

$$
\sigma(B(u, r / L))-\sigma\left(\bigcup_{v \in V_{b}(u)} B(v, \epsilon / L)\right) \geq \frac{\varrho}{2} \sigma(B(u, r / L))
$$

so using (17) and (16), we get

$$
\frac{\varrho}{2} \sigma(B(u, r / 2 L)) \leq \sigma\left(E_{L}^{*} \cap B(u, r / L)\right),
$$

and thus, $E_{L}^{*}$ is relatively dense.

Applying our unweighted result Theorem 3.1 to $E_{L}^{*}$ and to $M_{2 L} \in \Pi_{2 L}$, such that $M_{2 L}=Q_{L} R_{L}$ in $\mathbb{S}^{d}$, we get

$$
\sup _{u \in \mathbb{S}^{d}}\left|Q_{L}(u)\right| \omega(u) \lesssim \sup _{u \in E_{L}^{*}}\left|Q_{L}(u)\right| \omega_{L}(u), \quad Q_{L} \in \Pi_{L}
$$

We can take $\epsilon>0$ small enough, so that spherical harmonics of degree $\leq L$ are pointwise equivalents in spherical caps of radius $\epsilon / L$ where they reach their maximum. Indeed, all we have to do is to apply Bernstein's inequality, as we did in proving Proposition 2.1. 
Let $w \in B(v, \epsilon / L)$, with $v$ the center of a cap in $E_{L}^{*}$. We apply the $A_{\infty}$ condition, getting

$$
\begin{aligned}
\omega_{L}(w) & =\frac{1}{\sigma(B(w, 1 / L))} \int_{B(w, 1 / L)} \omega(u) d \sigma(u) \\
& \leq \frac{K}{\sigma(B(w, 1 / L))}\left(\frac{\sigma(B(w, 1 / L))}{\sigma\left(B(v, \epsilon / L) \cap E_{L}\right)}\right)^{s} \int_{B(v, \epsilon / L) \cap E_{L}} \omega(u) d \sigma(u) \\
& \leq \frac{C}{\sigma(B(w, 1 / L))}\left(\frac{\sigma(B(w, 1 / L))}{\delta \sigma(B(v, \epsilon / L))}\right)^{s} \int_{B(v, \epsilon / L) \cap E_{L}} \omega(u) d \sigma(u) \\
& =C_{\epsilon, \delta} L^{d} \int_{B(v, \epsilon / L) \cap E_{L}} \omega(u) d \sigma(u) .
\end{aligned}
$$

Finally, there exists $u \in V_{g}$, such that

$$
\sup _{u \in E_{L}^{*}}\left|Q_{L}(u)\right| \omega_{L}(u)=\sup _{u \in B(v, \epsilon / L)}\left|O_{L}(u)\right| \omega_{L}(u),
$$

for any $w \in B(v, \epsilon / L)$,

$$
\begin{aligned}
\inf _{u \in B(v, \epsilon / L)}\left|Q_{L}(u)\right| \omega_{L}(w) & \leq L^{d} \inf _{u \in B(v, \epsilon / L)}\left|Q_{L}(u)\right| \int_{B(v, \epsilon / L) \cap E_{L}} \omega(z) d \sigma(z) \\
& \leq \sup _{u \in B(v, \epsilon / L) \cap E_{L}}\left|Q_{L}(u)\right| \omega(u),
\end{aligned}
$$

and the result follows easily.

\section{Acknowledgment}

The authors would like to thank Konstantin Dyakonov and Salvador Rodríguez for pointing out some references and the anonymous referee for useful remarks.

\section{Funding}

This work was supported by projects MTM2005-08984-C02-02 and 2005SGR00611.

\section{References}

[1] Cruz-Uribe, D., and C. J. Neugebauer. "The structure of the reverse Hölder classes." Transactions of the American Mathematical Society 347, no. 8 (1995): 2941-60.

[2] Dai, F. "Multivariate polynomial inequalities with respect to doubling weights and $A_{\infty}$ weights." Journal of Functional Analysis 235, no. 1 (2006): 137-70. 
[3] Fefferman, C., and B. Muckenhoupt. "Two nonequivalent conditions for weight functions." Proceedings of the American Mathematical Society 45 (1974): 99-104.

[4] Havin, V., and B. Joricke. The Uncertainty Principle in Harmonic Analysis. Berlin: Springer, 1994.

[5] Logvinenko, V. N., and Ju. F. Sereda. "Equivalent norms in spaces of entire functions of exponential type." Teor. funktsii, funkt. analiz i ich prilozhenia 20 (1974): 102-11, 175.

[6] Luecking, D. H. "Equivalent norms on $L^{p}$ spaces of harmonic functions." Monatshefte fur Mathematik 96, no. 2 (1983): 133-41.

[7] Marzo, J. "Marcinkiewicz-Zygmund inequalities and interpolation by spherical harmonics." Journal of Functional Analysis 250, no. 2 (2007): 559-87.

[8] Mastroianni, G., and V. Totik. "Weighted polynomial inequalities with doubling and $A_{\infty}$ weights." Constructive Approximation 16, no. 1 (2000): 37-71.

[9] Ortega-Cerdà, J., and J. Saludes. "Marcinkiewicz-Zygmund inequalities." Journal of Approximation Theory 145, no. 2 (2007): 237-52.

[10] Stein, E. M. "Harmonic analysis: Real-variable methods, orthogonality, and oscillatory integrals." In Monographs in Harmonic Analysis. Vol. 3. Princeton Mathematical Series 43. Princeton, NJ: Princeton University Press, 1993.

[11] Szegö, G. Orthogonal Polynomials. Colloquium Publications 23. Providence, RI: American Mathematical Society, 1991.

[12] Volberg, A. L. "Thin and thick families of rational fractions." In Complex Analysis and Spectral Theory, 440-80. Lecture Notes in Mathematics 864. Berlin: Springer, 1981. 